\title{
Article
}

\section{Volatility Spillovers among Cryptocurrencies}

\author{
Lee A. Smales
}

UWA Business School, University of Western Australia, Perth, WA 6009, Australia; lee.smales@uwa.edu.au

\begin{abstract}
The cryptocurrency market has experienced stunning growth, with market value exceeding USD 1.5 trillion. We use a DCC-MGARCH model to examine the return and volatility spillovers across three distinct classes of cryptocurrencies: coins, tokens, and stablecoins. Our results demonstrate that conditional correlations are time-varying, peaking during the COVID-19 pandemic sell-off of March 2020, and that both ARCH and GARCH effects play an important role in determining conditional volatility among cryptocurrencies. We find a bi-directional relationship for returns and long-term (GARCH) spillovers between BTC and ETH, but only a unidirectional short-term (ARCH) spillover effect from BTC to ETH. We also find spillovers from BTC and ETH to USDT, but no influence running in the other direction. Our results suggest that USDT does not currently play an important role in volatility transmission across cryptocurrency markets. We also demonstrate applications of our results to hedging and optimal portfolio construction.
\end{abstract}

Keywords: cryptocurrency; volatility spillovers; vector autoregression (VAR); bitcoin; multivariate GARCH (MGARCH)

\section{JEL Classification: G10; G15; G23; C32}

Citation: Smales, Lee A. 2021.

Volatility Spillovers among Cryptocurrencies. Journal of Risk and Financial Management 14: 493. https://doi.org/10.3390/jrfm14100493

Academic Editors: Thanasis Stengos and Shigeyuki Hamori

Received: 14 September 2021

Accepted: 13 October 2021

Published: 15 October 2021

Publisher's Note: MDPI stays neutral with regard to jurisdictional claims in published maps and institutional affiliations.

Copyright: (C) 2021 by the author. Licensee MDPI, Basel, Switzerland. This article is an open access article distributed under the terms and conditions of the Creative Commons Attribution (CC BY) license (https:// creativecommons.org/licenses/by/ $4.0 /)$.

\section{Introduction}

Since the emergence of Bitcoin in 2009, the cryptocurrency market has experienced exponential growth. As of June 2021, over 4000 cryptocurrencies, with a total market value exceeding USD 1.5 trillion, are in existence ${ }^{1}$. With the initial stage of market development dominated by retail investors, the increasing relevance and comparative maturity of the crypto market has also attracted institutional investors. Each of the cryptocurrencies has specific attributes, and can broadly be classified as coins, tokens, or stablecoins. Since investors potentially have interest in diversifying their crypto holdings across different classes of cryptocurrencies, to enable effective risk management, it is important to develop a better understanding of their interconnectedness, and how shocks transmit from one cryptocurrency to another.

Our study considers the return and volatility spillovers across three distinct classes of cryptocurrencies. Bitcoin (BTC) is an example of a cryptocurrency coin as it functions as a currency within its own blockchain ${ }^{2}$; it is the original and largest cryptocurrency, with market capitalisation of USD $650 \mathrm{bn}+{ }^{3}$. Ether $(E T H)$ is a transactional token that facilitates operations on the Ethereum blockchain and is the second largest cryptocurrency (USD $265 \mathrm{bn}+)$. The Bitcoin blockchain is limited in that it only facilitates transactions involving Bitcoin. In contrast, the Ethereum blockchain allows for the creation of smart contracts and plays a key role in decentralised finance (DeFi). Finally, Tether (USDT) is a stablecoin, an altcoin that is backed by real-world assets (cash and short-term money-market instruments) with the intention of making them less volatile.

Reflecting their novelty and absence of intrinsic value, cryptocurrency returns are more volatile, with heavier tails, than those of traditional investments (Gkillas and Katsiampa (2018); Smales (2019); Härdle et al. (2020); Liu and Tsyvinski (2021)). In his survey, Kyriazis (2019) notes that the literature has investigated spillovers between crypto and other assets (Bouri et al. (2018)) as well as among crypto markets; we focus on the latter. 
As the cryptocurrency market has matured, volatility spillovers have trended higher in recent years (Koutmos (2018); Yi et al. (2018); Kumar and Anandarao (2019); Xu et al. (2021)), indicating a greater level of connectedness, and underlining the limit to diversification within cryptocurrency markets. While Moratis (2021) argue that size is not the only determinant of spillover effects, several studies find that Bitcoin, the largest cryptocurrency with at least $40 \%$ of total market value, has the greatest influence on fluctuations in other cryptocurrencies (Koutmos (2018); Kyriazis (2019); Kumar and Anandarao (2019); Smales (2020)) and sits at the centre of a connected network (Ji et al. (2019)). The second largest cryptocurrency, Ether, is also shown to be relevant for volatility spillovers and acts as a "connection hub" for other cryptocurrencies (Sensoy et al. (2021); Xu et al. (2021)). Linkages seem to differ between the short- and long-run (Qureshi et al. (2020)) including for unexpected volatility, or volatility surprises (Bouri et al. (2020)).

More recently, the literature has demonstrated that connectedness increases during cyber-attacks (Caporale et al. (2021)) and extreme events (Bouri et al. (2021b)). Bouri et al. (2021a) suggest that changes in connectedness may be linked to the "happiness" of investors, with higher connectedness and greater market volatility when investors are unhappy.

Our study is most related to those of Beneki et al. (2019), Katsiampa et al. (2019), and Canh et al. (2019). All find that time-varying conditional correlations occur between cryptocurrencies. The first two employ bivariate BEKK models, with Katsiampa et al. (2019) identifying bi-directional volatility spillover between Bitcoin and Ether, while Beneki et al. (2019) find only uni-direction transmission from Ether to Bitcoin. Canh et al. (2019) utilise a DCC-MGARCH specification that is similar to our preferred model and demonstrate the existence of strong positive correlations and volatility spillovers.

Our empirical results demonstrate that both ARCH and GARCH effects play an important role in determining conditional volatility among cryptocurrencies. We find a bi-directional relationship for returns and long-term (GARCH) spillovers between BTC and $\mathrm{ETH}$, but only a unidirectional short-term (ARCH) spillover effect from BTC to ETH. We also find spillovers from BTC and ETH to USDT, but no influence running in the other direction, suggesting that USDT does not play an important role in volatility transmission across cryptocurrency markets. The indicated relationships are similar across a set of MGARCH models. Consistent with the literature, our results also demonstrate that the conditional correlation is time-varying. Our choice of sample period allows us to illustrate that the correlation peaks in March 2020, a period associated with a COVID-19-induced crash in the prices of cryptocurrencies and other financial instruments.

We consider spillovers across three different classes of cryptocurrencies and are the first to consider a stablecoin within this setting. Our results have implications for investors seeking optimal portfolio decisions. In addition, to the extent that spillover patterns offer a measure of contagion risk (Koutmos (2018)) they have connotations for policy-makers watchful for systemic risk.

The rest of the paper proceeds as follows: Section 2 outlines the cryptocurrency data used, highlighting unconditional volatility and correlation. Section 3 provides the empirical analysis, including brief discussion of potential applications. Section 4 concludes.

\section{Data}

This study utilises a series of daily returns for Bitcoin (BTC), Ether $(E T H)$, and Tether (USDT) cryptocurrencies for a sample period running from 1-Janary-2016 to 30-June-2021. Returns for cryptocurrency $i$, on day $t$, are calculated as $r_{i t}=\ln \left(p_{i t} / p_{i t-1}\right)$, using daily closing prices obtained from www.coinmarketcap.com (accessed on 12 September 2021). The sample period is chosen to coincide with data availability ${ }^{4}$.

Figure 1 plots the time series of closing prices and market values during our sample period. Coinciding with a rise in the price of a Bitcoin from USD 434 to USD 35,041, the market value of Bitcoin has increased from USD 7 billion to USD 657 billion, having peaked at USD 1.1 trillion in April 2021. The Ether token price has increased even more quickly, 
from USD 0.95 to USD 2275, as the market value reached USD 265 billion. In contrast, the market value of Tether has reached USD 62 billion despite the price largely maintaining parity at USD 1. This illustrates the different nature of a stablecoin relative to other types of cryptocurrencies.

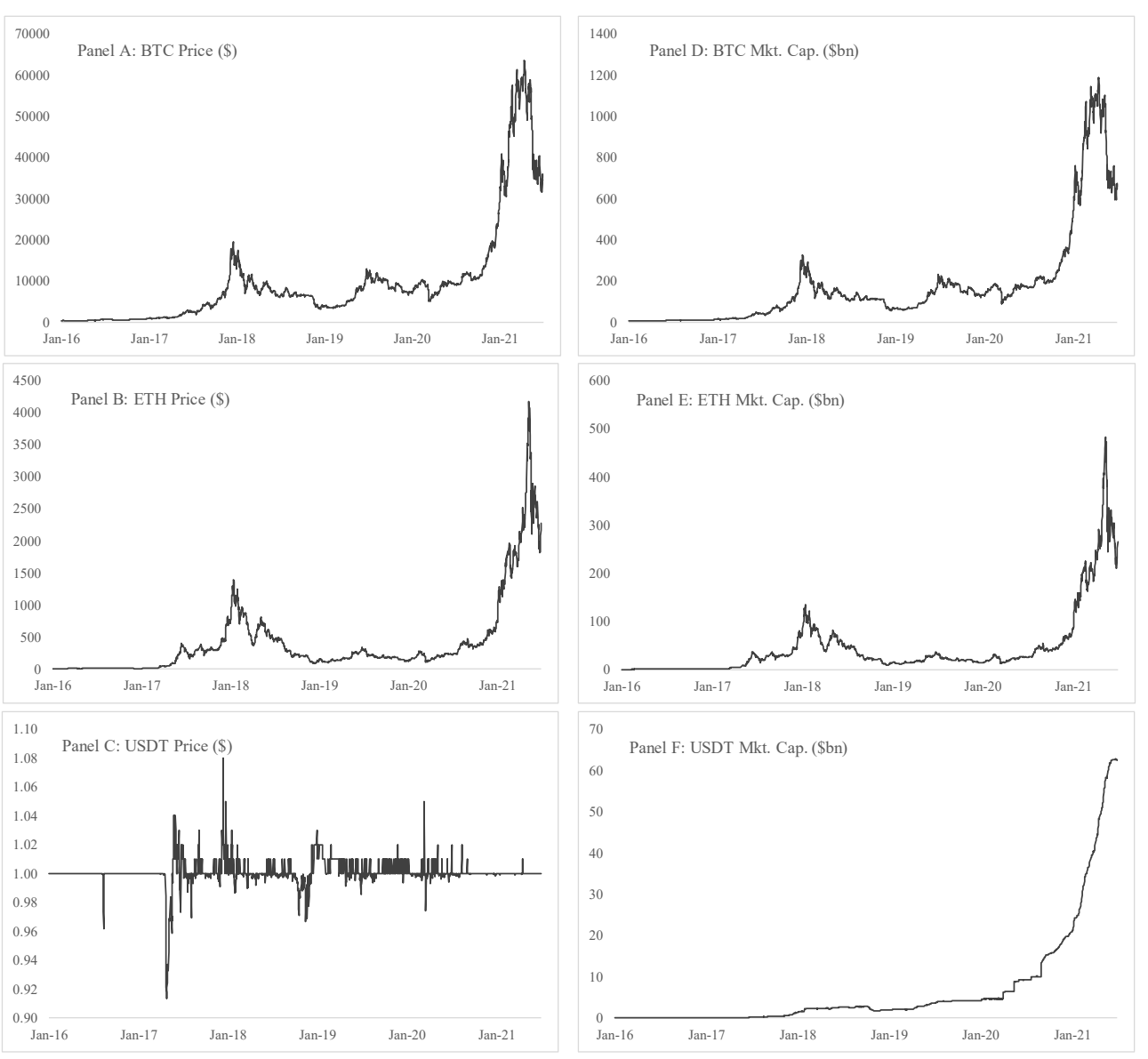

Figure 1. Cryptocurrency price and market capitalisation.

Summary statistics for the series of daily returns are shown in Table 1 . Ether has the largest $(\mu=0.39 \%)$ and most volatile $(\sigma=6.00 \%)$ returns. The largest daily fall is $55.1 \%$ on 12-March-2020. As expected, Tether's returns are very stable, although there is still a minimum of $-4.9 \%$ (13-March-2020) and a maximum of $+5.7 \%$. All cryptocurrencies exhibit a high degree of kurtosis ("fat-tails") that is common among financial instruments.

Table 1. Summary statistics for daily returns.

\begin{tabular}{cccc}
\hline & BTC & ETH & USDT \\
\hline Mean & 0.0022 & 0.0039 & 0.0000 \\
Median & 0.0021 & 0.0009 & 0.0000 \\
Std. Dev. & 0.040 & 0.060 & 0.006 \\
Maximum & 0.225 & 0.303 & 0.057 \\
Minimum & -0.465 & -0.551 & -0.049 \\
Skewness & -0.795 & -0.284 & 0.293 \\
Kurtosis & 14.96 & 10.48 & 20.76 \\
Observations & 2007 & 2007 & 2007
\end{tabular}

Note: This table provides summary statistics for daily returns of the cryptocurrencies used in our study; Bitcoin (BTC), Ether (ETH), and Tether (USDT). Sample period: 1-Jan-2016 to 30-June-2021. 
Table 2 shows that there is a high degree of positive correlation between BTC and ETH returns. Rolling 365-day correlations (Figure 2) show that this correlation has increased markedly over time, as the crypto market has matured, and has remained above 0.7 since late 2018. In contrast, USDT has a low level of negative correlation with BTC and ETH. In both cases, the rolling correlation increased from the start of the sample, reaching a peak close to +0.3 in October 2019, dropped sharply in March 2020, and reached a low of -0.3 in December 2020.

Table 2. Correlations between returns and squared returns.

\begin{tabular}{cccc}
\hline & BTC & ETH & USDT \\
\hline BTC & 1.000 & $\mathbf{0 . 6 6 8}$ & $\mathbf{0 . 3 4 1}$ \\
ETH & $\mathbf{0 . 5 8 1}$ & 1.000 & $\mathbf{0 . 3 1 9}$ \\
USDT & -0.015 & -0.017 & 1.000 \\
\hline
\end{tabular}

Note: This table provides correlation for daily returns (bottom left) and squared returns (top right) for the cryptocurrencies used in our study. Significance at $1 \%$ level indicated by bold.

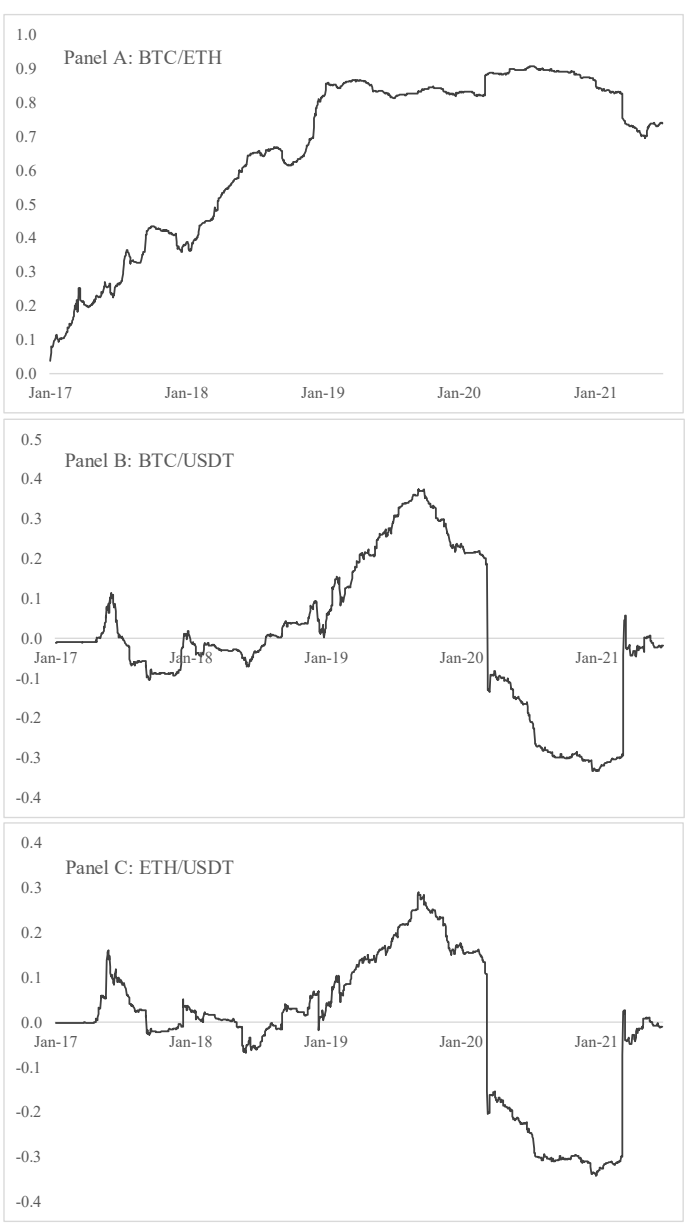

Figure 2. Rolling unconditional correlation (1 year).

Figure 3 plots the times series of squared returns and illustrates that volatility exhibits clustering and has varied considerably over time. Although the magnitude of volatility is significantly lower for USDT (the figure multiplies USDT squared returns by 100), all three cryptocurrencies display similar patterns, and have significant positive correlation (Table 2). There are clusters of volatility from mid-2017 to early 2018 and in early 2021 (both consistent with a sharp rise and subsequent fall in cryptocurrency prices at those times) along with a 
large spike in March 2020. ARCH-LM tests (Engle 1982), test the null hypothesis that there is no ARCH up to order five in the residuals using:

$$
\varepsilon_{t}^{2}=\beta_{0}+\left(\sum_{s=1}^{5} \beta_{s} \varepsilon_{t-s}^{2}\right)+v_{t}
$$

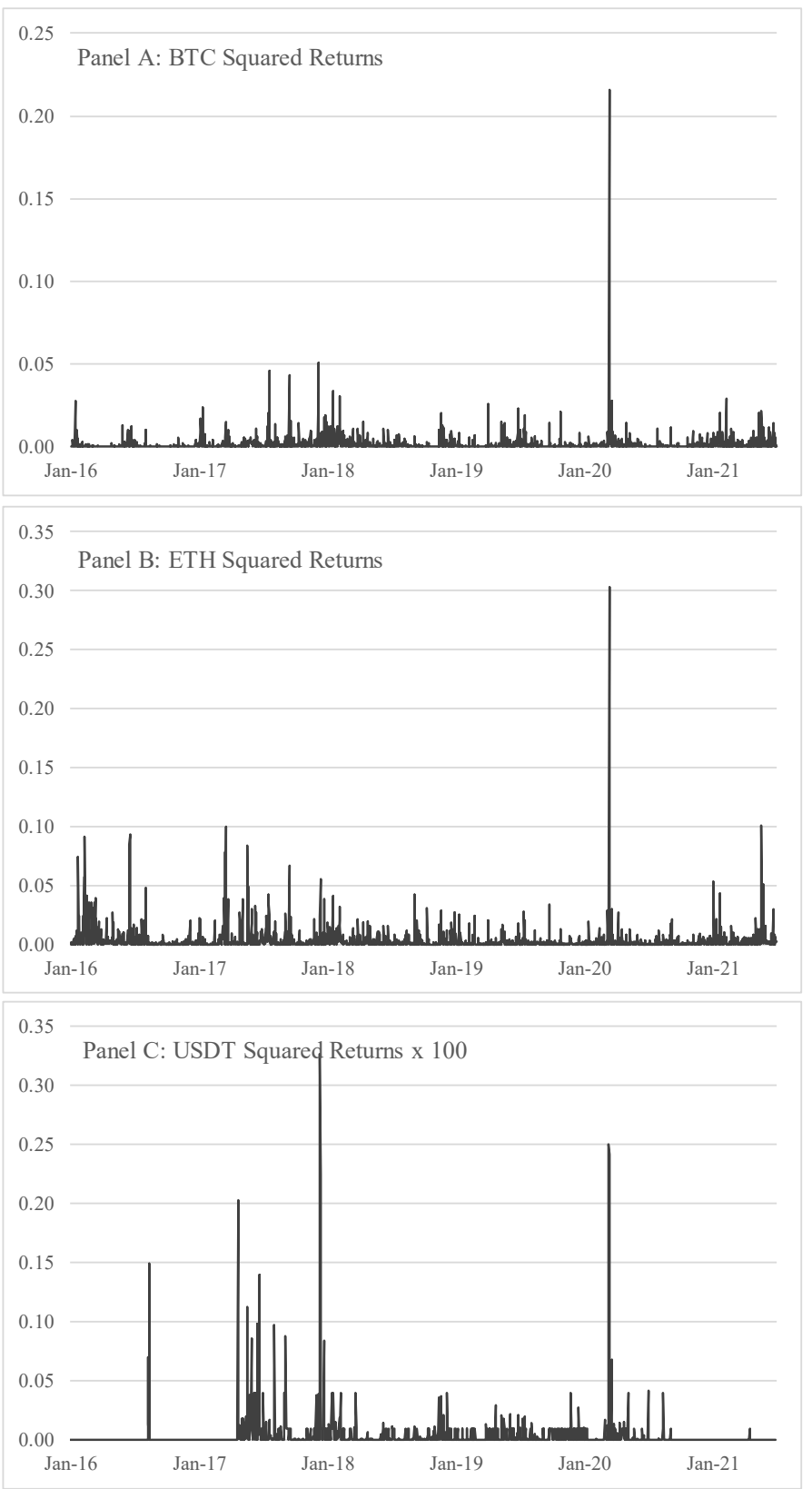

Figure 3. Cryptocurrency squared returns.

Table 3 shows that we cannot reject the null hypothesis for the return series in any of our cryptocurrencies. This is consistent with the evidence of long memory in Bitcoin volatility noted by Bouri et al. (2019) and aids justification of the use of GARCH-based models.

Together, this introductory analysis suggests that it is worthwhile considering ARCH/ GARCH type models that incorporate dynamic correlations and time-varying volatility. 
Table 3. ARCH-LM Test.

\begin{tabular}{cccc}
\hline ARCH-LM Test & & & \\
\hline BTC Returns & & & \\
F-Statistic & 0.259 & Prob. & 0.979 \\
Obs $\times$ R-squared & 2.077 & Prob. & 0.979 \\
ETH Returns & & & \\
F-Statistic & 0.798 & Prob. & 0.589 \\
Obs $\times$ R-squared & 5.591 & Prob. & 0.588 \\
USDT Returns & & & \\
F-Statistic & 1.191 & Prob. & 0.275 \\
Obs $\times$ R-squared & 1.192 & Prob. & 0.275
\end{tabular}

Note: This table reports the results for ARCH-LM Test assessed using Equation (1) with 5 lags. Obs $\times$ R-squared is Engle's LM test statistic.

\section{Empirical Analysis}

Our empirical analysis focuses on four multivariate GARCH (MGARCH) models to examine the volatility dynamics between cryptocurrencies. In a similar vein to Sadorsky (2012), we use three different specifications-BEKK, constant conditional correlation (CCC), and dynamic conditional correlation (DCC). While our initial analysis suggests that Engle's (2002) DCC model may be the most appropriate model type for our data, we include the others to allow for model comparison and robustness. Returns are modelled using a vector autoregression (VAR) with one lag, while time-varying variances and covariances are captured by MGARCH models. To allow for volatility spillovers, we assume the conditional variance of the diagonal, CCC, and DCC models follows a VARMA-GARCH $(1,1)$ typical of Ling and McAleer (2003). This permits the identification of the causality direction without ex ante assumptions on direction. We specify the models as:

$$
\begin{gathered}
r_{i t}=m_{i 0}+\sum_{j=1}^{3} m_{i j} r_{j t-1}+\varepsilon_{i t}, \varepsilon_{i t} \mid I_{i t-1} \sim N\left(0, h_{t}\right), i=1,2,3 \\
\varepsilon_{t}=v_{i t} h_{i t}^{0.5}, v_{i t} \sim N(0,1) \\
h_{i t}=c_{i j}+\sum_{j=1}^{3} \alpha_{i j} \varepsilon_{j t-1}^{2}+\sum_{j=1}^{3} \beta_{i j} h_{j t-1}
\end{gathered}
$$

where $r_{i t}$ is the return for cryptocurrency $i$ on day $t$ and $\varepsilon_{i t}$ is a random error term with conditional variance $h_{i t} . I_{i t-1}$ denotes the market information available at time $t-1$. Equation (3) specifies the relationship between the error term $\varepsilon_{i t}$ and the conditional variance $h_{i t}$. Equation (4) models the conditional variance, $h_{i t}$, as a VARMA-GARCH $(1,1)$ process. The models are estimated by quasi-maximum likelihood estimation (QMLE) with the BFGS algorithm and robust standard errors are reported.

Table 4 reports the estimated coefficients with the returns series for Bitcoin prices (BTC) identified by $i=1$, the return series for Ether prices $(E T H)$ identified by $i=2$, and the return series for Tether prices (USDT) identified by $i=3$.

The preferred model, selected on the basis of AIC and log likelihood, is the DCC model. This is consistent with the time-varying volatility (Figure 3) and dynamic correlations (Figure 2) indicated earlier. Our discussion therefore focuses on this model, but differences and/or similarities with the other specifications are indicated when important. 
Table 4. MGARCH parameter estimates.

\begin{tabular}{|c|c|c|c|c|c|c|c|c|c|c|c|c|}
\hline & \multicolumn{2}{|c|}{ BEKK } & \multicolumn{4}{|c|}{$\mathrm{CCC}$} & \multicolumn{2}{|c|}{ DCC } & \multicolumn{4}{|c|}{ DCC (BTC/ETH) } \\
\hline & Coeff. & Std.Err. & & Coeff. & Std.Err. & & Coeff. & Std.Err. & & Coeff. & Std.Err. & \\
\hline \multicolumn{13}{|l|}{ Mean } \\
\hline $\mathrm{m}_{10}$ & 0.002 & 0.001 & $* * *$ & 0.001 & 0.000 & $* *$ & 0.000 & 0.000 & $* * *$ & 0.000 & 0.000 & \\
\hline $\mathrm{m}_{11}$ & 0.044 & 0.023 & $*$ & 0.950 & 0.009 & $* * *$ & 0.862 & 0.000 & $* * *$ & 0.989 & 0.000 & $* * *$ \\
\hline $\mathrm{m}_{12}$ & -0.039 & 0.014 & $* * *$ & -0.061 & 0.013 & $* * *$ & -0.016 & 0.000 & $* * *$ & 0.000 & 0.000 & $* * *$ \\
\hline $\mathrm{m}_{13}$ & 0.174 & 0.179 & & -0.024 & 0.263 & & -0.038 & 0.002 & $* * *$ & & & \\
\hline $\mathrm{m}_{20}$ & 0.002 & 0.001 & $* *$ & 0.004 & 0.002 & $* *$ & 0.001 & 0.000 & $* * *$ & 0.002 & 0.001 & * \\
\hline $\mathrm{m}_{21}$ & -0.082 & 0.041 & $* *$ & -0.068 & 0.017 & $* * *$ & -0.004 & 0.000 & $* * *$ & -0.112 & 0.022 & $* * *$ \\
\hline $\mathrm{m}_{22}$ & 0.045 & 0.040 & & 1.027 & 0.008 & $* * *$ & 0.003 & 0.000 & $* * *$ & 0.139 & 0.018 & $* * *$ \\
\hline $\mathrm{m}_{23}$ & 0.343 & 0.228 & & -0.028 & 0.267 & & -0.008 & 0.003 & $* * *$ & & & \\
\hline $\mathrm{m}_{30}$ & 0.000 & 0.000 & & 0.000 & 0.000 & & 0.000 & 0.000 & & & & \\
\hline $\mathrm{m}_{31}$ & 0.000 & 0.001 & & 0.001 & 0.000 & $* *$ & 0.000 & 0.000 & & & & \\
\hline$m_{32}$ & -0.003 & 0.001 & $* *$ & 0.000 & 0.000 & & 0.000 & 0.002 & & & & \\
\hline $\mathrm{m}_{33}$ & -0.476 & 0.050 & $* * *$ & 0.045 & 0.271 & & 1.000 & 0.004 & $* * *$ & & & \\
\hline \multicolumn{13}{|l|}{ Variance } \\
\hline $\mathrm{c}_{11}$ & 0.009 & 0.002 & $* * *$ & 0.001 & 0.000 & $* * *$ & 0.001 & 0.000 & $* * *$ & 0.000 & 0.000 & $* * *$ \\
\hline$c_{21}$ & 0.014 & 0.004 & $* * *$ & & & & & & & & & \\
\hline $\mathrm{c}_{22}$ & 0.009 & 0.002 & $* * *$ & 0.002 & 0.000 & $* * *$ & 0.002 & 0.000 & $* * *$ & 0.002 & 0.000 & $* * *$ \\
\hline$c_{31}$ & 0.000 & 0.000 & * & & & & & & & & & \\
\hline$c_{32}$ & 0.000 & 0.000 & * & & & & & & & & & \\
\hline$c_{33}$ & 0.000 & 0.000 & & 0.000 & 0.000 & $* * *$ & 0.000 & 0.000 & $* * *$ & & & \\
\hline$\alpha_{11}$ & 0.405 & 0.063 & $* * *$ & 0.073 & 0.110 & & 0.041 & 0.000 & $* * *$ & 0.020 & 0.191 & \\
\hline$\alpha_{12}$ & -0.096 & 0.128 & & -0.003 & 0.107 & & -0.005 & 0.005 & & 0.000 & 0.000 & \\
\hline$\alpha_{13}$ & -0.007 & 0.003 & $* *$ & -0.276 & 2.600 & & -0.013 & 15.851 & & & & \\
\hline$\alpha_{21}$ & -0.021 & 0.023 & & -0.015 & 0.173 & & -0.050 & 0.005 & $* * *$ & -0.048 & 0.013 & $* * *$ \\
\hline$\alpha_{22}$ & 0.469 & 0.105 & $* * *$ & 0.073 & 0.161 & & 0.063 & 0.000 & $* * *$ & 0.055 & 0.008 & $* * *$ \\
\hline$\alpha_{23}$ & -0.004 & 0.002 & * & -0.483 & 3.815 & & -0.015 & 13.421 & & & & \\
\hline$\alpha_{31}$ & 0.061 & 0.249 & & 0.001 & 0.001 & & 0.002 & 0.000 & $* * *$ & & & \\
\hline$\alpha_{32}$ & 0.207 & 0.329 & & 0.000 & 0.001 & & 0.000 & 0.000 & $* * *$ & & & \\
\hline$\alpha_{33}$ & 0.460 & 0.080 & $* * *$ & 0.670 & 0.264 & & 0.260 & 1.232 & & & & \\
\hline$\beta_{11}$ & 0.907 & 0.026 & $* * *$ & 0.442 & 0.002 & $* * *$ & 0.464 & 0.000 & $* * *$ & 0.106 & 0.043 & $* *$ \\
\hline$\beta_{12}$ & -0.013 & 0.055 & & -0.003 & 0.001 & $* * *$ & -0.024 & 0.000 & $* * *$ & -0.001 & 0.000 & $* * *$ \\
\hline$\beta_{13}$ & 0.002 & 0.001 & * & 0.083 & 0.645 & & 0.019 & 0.078 & & & & \\
\hline$\beta_{21}$ & 0.000 & 0.015 & & 0.012 & 0.002 & $* * *$ & 0.060 & 0.000 & $* * *$ & 0.033 & 1.122 & \\
\hline$\beta_{22}$ & 0.867 & 0.062 & $* * *$ & 0.444 & 0.001 & $* * *$ & 0.505 & 0.000 & $* * *$ & 0.525 & 0.000 & $* * *$ \\
\hline$\beta_{23}$ & -0.003 & 0.001 & $*$ & -0.131 & 1.102 & & -0.019 & 0.025 & & & & \\
\hline$\beta_{31}$ & -0.051 & 0.062 & & -0.004 & 0.000 & $* * *$ & -0.008 & 0.000 & $* * *$ & & & \\
\hline$\beta_{32}$ & -0.087 & 0.096 & & -0.003 & 0.000 & $* * *$ & -0.001 & 0.000 & $* * *$ & & & \\
\hline$\beta_{33}$ & 0.938 & 0.012 & $* * *$ & 0.578 & 0.015 & $* * *$ & 0.479 & 0.000 & $* * *$ & & & \\
\hline$\varrho_{21}$ & & & & 0.303 & 0.374 & & & & & & & \\
\hline$\varrho_{31}$ & & & & -0.010 & 0.087 & & & & & & & \\
\hline$Q_{32}$ & & & & -0.038 & 0.133 & & & & & & & \\
\hline$\theta_{1}$ & & & & & & & 0.102 & 0.057 & * & 0.168 & 0.036 & $* * *$ \\
\hline$\theta_{2}$ & & & & & & & 0.693 & 0.146 & $* * *$ & 0.809 & 0.039 & $* * *$ \\
\hline $\log L$ & \multicolumn{2}{|c|}{26141} & \multicolumn{4}{|c|}{26369} & \multicolumn{2}{|c|}{24555} & \multicolumn{4}{|c|}{24205} \\
\hline AIC & \multicolumn{2}{|c|}{-26.06} & \multicolumn{4}{|c|}{-26.24} & \multicolumn{2}{|c|}{-24.43} & \multicolumn{4}{|c|}{-24.10} \\
\hline
\end{tabular}

Note: Models are estimated using QMLE with robust standard errors. Variable order is BTC (1), ETH (2), and USDT (3) returns. In the variance equations, $\mathrm{c}$ denotes the constant terms, $\alpha$ denotes the ARCH terms, and $\beta$ denotes the GARCH terms. In the mean equation $\mathrm{m}_{13}$ represents the effect of a one period lag USDT returns on current period BTC returns. In the variance equation, $\alpha_{13}$ represents the short-term volatility spillover from USDT to BTC while $\beta_{13}$ represents the long-term volatility spillover from USDT to BTC. There are 2007 observations and the sample period runs from January 2016 to June $2021 .{ }^{*}, * *$, and ${ }^{* * *}$ indicate statistical significance at the $10 \%, 5 \%$, and $1 \%$ level respectively.

The mean (return) equation is considered first. There is significant positive own correlation of returns for BTC $\left(\mathrm{m}_{11}\right)$ and ETH $\left(\mathrm{m}_{22}\right)$, indicating that cryptocurrency prices tend to trend, whereas the picture is less clear for the USDT $\left(\mathrm{m}_{33}\right)$ stablecoin. There is also evidence across models that indicates Bitcoin returns are negatively influenced by 
prior period Ether returns $\left(\mathrm{m}_{12}\right)$ and vice versa $\left(\mathrm{m}_{21}\right)$. It also appears that prior period Tether returns negatively impact Bitcoin $\left(\mathrm{m}_{13}\right)$ and Ether returns $\left(\mathrm{m}_{23}\right)$, but this effect is not reciprocated.

Both ARCH and GARCH effects appear to play an important role in determining conditional volatility. The own conditional ARCH effects provide an indication of shortterm volatility persistence. In this case, they are positive and highly significant for BTC $\left(\alpha_{11}\right)$ and ETH $\left(\alpha_{22}\right)$ and positive but insignificant for USDT $\left(\alpha_{33}\right)$, although the latter is also significant in the BEKK and CCC model. Long-term volatility persistence effects, indicated by own conditional GARCH, are also positive and highly significant for BTC $\left(\beta_{11}\right)$, ETH $\left(\beta_{22}\right)$, and USDT $\left(\beta_{33}\right)$, with each displaying a similar level of persistence (indicated by the magnitude of the coefficient). Since $\alpha_{11}<\beta_{11}, \alpha_{22}<\beta_{22}$, and $\alpha_{33}<\beta_{33}$ short-run volatility persistence $(\mathrm{ARCH})$ is lower than long-run persistence (GARCH) for all three cryptocurrencies.

The DCC model also provides evidence of significant volatility spillovers from one cryptocurrency to another. While the statistical significance varies across the other models, the indicated direction is consistent. There is unidirectional short-term spillover from BTC to ETH $\left(\alpha_{21}\right)$ and bi-directional long-term spillovers between BTC and ETH $\left(\beta_{21}, \beta_{12}\right)$. Although not all of the spillover signs are positive, the existence of bi-directional spillovers is consistent with Katsiampa et al. (2019) and Canh et al. (2019). There is also evidence of both short- and long-term unidirectional spillovers from BTC to USDT $\left(\alpha_{31}, \beta_{31}\right)$ and from ETH to USDT $\left(\alpha_{32}, \beta_{32}\right)$. We identify no short-term or long-term volatility spillovers from USDT to either of the other cryptocurrencies. This suggests that USDT does not play an important role in volatility transmission across cryptocurrency markets. Finally, the estimated $\theta_{1}$ and $\theta_{2}$ coefficients for the DCC model are positive and statistically significant at the $1 \%$ level. Since $\left(\theta_{1}+\theta_{2}\right)<1$, the dynamic conditional correlations are mean-reverting.

Table 5 shows displays the diagnostic tests for the standardised residuals and standardised residuals squared. For the preferred DCC model there is no evidence of serial correlation for BTC or ETH, but we cannot reject this for USDT.

Table 5. Diagnostic tests for standardised residuals.

\begin{tabular}{|c|c|c|c|c|c|c|c|c|c|}
\hline & \multicolumn{3}{|c|}{ BEKK } & \multicolumn{3}{|c|}{$\mathrm{CCC}$} & \multicolumn{3}{|c|}{$\mathrm{DCC}$} \\
\hline & BTC & ETH & USDT & BTC & ETH & USDT & BTC & ETH & USDT \\
\hline$Q(20) r$ & 25.810 & 45.380 & 35.450 & 67.670 & 31.990 & 6.766 & 201.170 & 47.640 & 14.116 \\
\hline$p$ values & 0.172 & 0.000 & 0.018 & 0.000 & 0.043 & 0.997 & 0.000 & 0.000 & 0.824 \\
\hline$Q(20) r^{2}$ & 5.150 & 14.790 & 4.997 & 808.790 & 135.590 & 0.309 & 361.950 & 67.580 & 1.694 \\
\hline$p$ values & 0.990 & 0.780 & 0.999 & 0.000 & 0.000 & 1.000 & 0.000 & 0.000 & 1.000 \\
\hline
\end{tabular}

Note: This table presents diagnostics tests for standardised residuals pertaining to each of the MGARCH models.

The time-varying conditional correlations related to the DCC model are depicted in Figure 4, with summary statistics in Table 6 Panel A. The average correlation between USDT and BTC $\left(\varrho_{13}\right)$ and ETH $\left(\varrho_{23}\right)$ is just -0.015 and, consistent with USDT's role as a stablecoin, does not vary significantly over time. Even the spike indicated in March 2020 is very small in economic terms. We also note the similarity with the low degree of unconditional correlation illustrated in Figure 2. In contrast, the dynamic conditional correlation between BTC and ETH $\left(\varrho_{12}\right)$ averages 0.53 and varies between 0.24 (Mar-2017) and 0.66 (Mar-2020) illustrating the need to compute dynamic conditional correlations. In all cases, conditional correlation peaks in March 2020, a period associated with a sharp decline in cryptocurrency (and other asset) prices. This is consistent with Bouri et al. (2021b) finding that connectedness increases during extreme events, and Naeem et al. (2021) showing that cryptocurrency markets were less efficient at the onset of the COVID19 outbreak. 


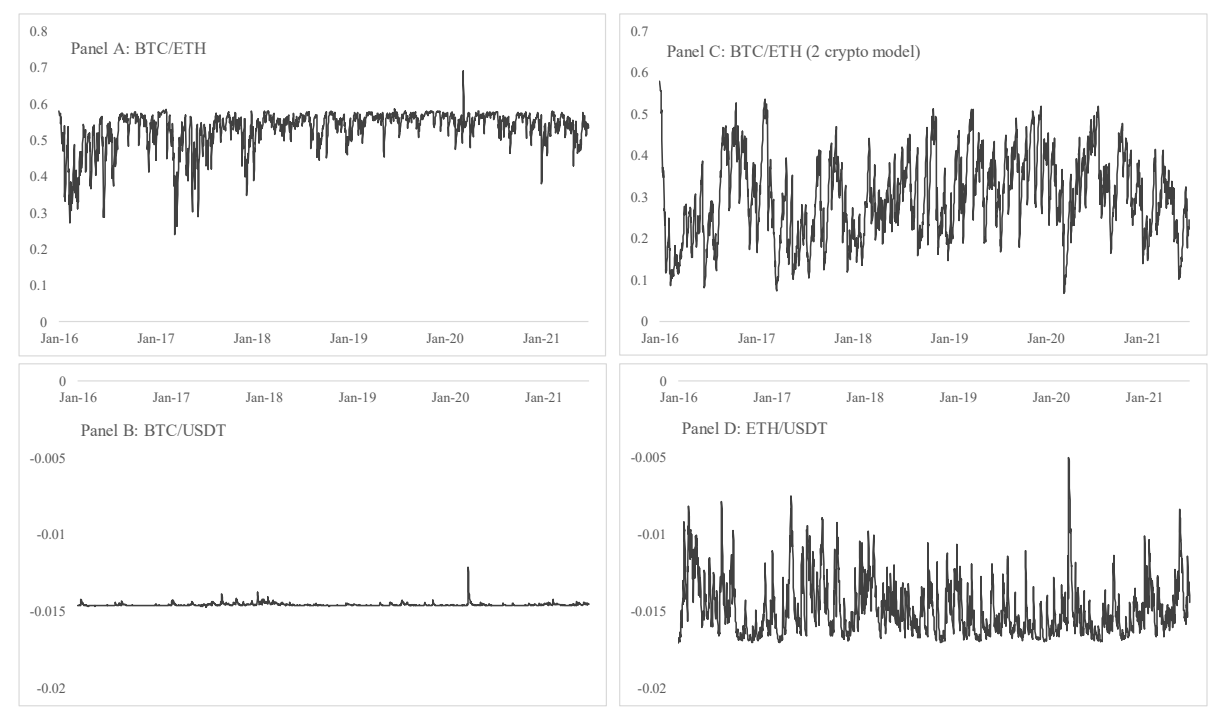

Figure 4. Dynamic conditional correlations (DCC Model).

Table 6. Dynamic correlation summary statistics.

\begin{tabular}{ccccc}
\hline & Mean & Std. Dev. & Min. & Max. \\
\hline & \multicolumn{2}{c}{ Panel A: Dynamic Conditional Correlation } \\
BTC/ETH & 0.531 & 0.053 & 0.239 & 0.690 \\
BTC/USDT & -0.015 & 0.000 & -0.015 & -0.012 \\
ETH/USDT & -0.015 & 0.002 & -0.017 & -0.005 \\
& \multicolumn{2}{c}{ Panel B: Hedge ratio (long/short) } \\
BTC/ETH & 0.955 & 0.088 & 0.638 & 2.676 \\
BTC/USDT & 0.000 & 0.000 & -0.008 & 0.000 \\
ETH/BTC & 0.297 & 0.047 & 0.006 & 0.355 \\
ETH/USDT & 0.000 & 0.000 & 0.000 & 0.000 \\
USDT/BTC & -1.226 & 0.916 & -7.263 & -0.002 \\
USDT/ETH & -2.279 & 1.762 & -10.072 & -0.093 \\
& \multicolumn{5}{c}{} \\
BTC/ETH & 0.022 & Panel C: Portfolio weights & 0.000 & 0.107 \\
BTC/USDT & 0.998 & 0.017 & 0.025 & 1.000 \\
ETH/USDT & 0.999 & 0.022 & 0.996 & 1.000 \\
\hline
\end{tabular}

Note: This table provides summary statistics for the dynamic correlation (Panel A) obtained from the DCC model and associated applications-hedge ratio (Panel B) and portfolio weights (Panel C).

Repeating the analysis with a DCC model that includes just BTC and ETH (last column of Table 5) provides for lower average (0.29), but still time-varying, conditional correlation. Regardless of which specification is used, the conditional correlation is lower than the rolling unconditional correlation shown in Figure 2.

It is important to place our results in the context of the existing literature, particularly in reference to work that has utilised MGARCH models to study the relationships between cryptocurrencies. Consistent with Beneki et al. (2019), Canh et al. (2019), and Katsiampa et al. (2019), we find time-varying conditional correlations between cryptocurrencies. While Canh et al. (2019) do not include ETH in their sample set, the correlation we find between BTC and ETH is consistent with the high correlations they note among other cryptocurrencies. Our results demonstrate a bi-directional relationship for returns and long-term spillovers between BTC and ETH, together with a unidirectional short-term spillover effect from BTC to ETH. This is congruent to the results of Katsiampa et al. (2019) and differs from that of Beneki et al. (2019) and Canh et al. (2019), who find a unidirectional effect running in the other direction (from ETH to BTC). No prior studies appear to have contemplated the volatility spillovers to and from stablecoins. 
Having identified dynamic conditional volatility estimates, we are able to utilise them in two potential applications: hedge ratios and optimal portfolio weights.

\subsection{Application 1: Hedge Ratios}

The dynamic conditional volatility estimates may be used to construct hedge ratios (Kroner and Sultan (1993); Sadorsky (2012)). For instance, a long position in cryptocurrency $i$ can be hedged with a short position in cryptocurrency $j$ using the hedge ratio:

$$
\beta_{i j, t}=h_{i j, t} / h_{j j, t}
$$

Summary statistics are reported in Table 6 Panel B. The low variance, and associated covariances, of USDT make interpretation of the hedge ratio results problematic, other than to say that USDT is likely not a good hedge for the other cryptocurrencies. Instead, we focus our discussion on BTC and ETH. The hedge ratio for BTC with ETH is close to one (0.96) and reasonably constant over time, with short-lived spikes in March 2017 and March 2020. At such times, when the hedge ratio goes above 1.0, it would be expensive to initiate the hedge. The hedge ratio for ETH with BTC is lower (0.30) but also remarkably constant over time (std. dev. of 0.05), with a minimum of 0.10 in March 2020, and a maximum of 0.36 in January 2016. Together, this suggests that BTC and ETH are suitable hedging instruments, but USDT is not (at least not in the context discussed here).

\subsection{Application 2: Portfolio Weights}

Using the methodology of Kroner and Ng (1998), Sadorsky (2012) shows optimal portfolio weights may be computed using the conditional volatility estimates of MGARCH models. For a portfolio of two assets (asset $i$, asset $j$ ), the weight of the first cryptocurrency at time $t$ is $w_{i j, t}, h_{j j, t}$ is the conditional variance of cryptocurrency $j$, and $h_{i j, t}$ is the conditional covariance between the two cryptocurrencies; the weights can be computed as:

$$
\begin{gathered}
w_{i j, t}=\frac{h_{j j, t}-h_{i j, t}}{h_{i i, t}-2 h_{i j, t}+h_{j j, t}} \\
w_{j i, t}=1-w_{i j, t} \\
w_{i j, t}=\left\{\begin{array}{c}
0, \text { if } w_{i j, t}<0 \\
w_{i j, t}, \text { if } 0 \leq w_{i j, t} \leq 1 \\
1, \text { if } w_{i j, t}>1
\end{array}\right.
\end{gathered}
$$

Table 6 Panel $C$ reports the summary statistics for portfolio weights. The average weight for the BTC/ETH portfolio is 0.022 , indicating that just USD 0.02 in every USD 1 portfolio should be invested in BTC, and USD 0.98 in ETH. The much higher weight attached to ETH is likely due to a combination of (a) ETH producing much higher returns over the sample period and (b) the relatively high correlation. Similarly, the stability of USDT leads to almost $100 \%$ allocation to BTC and ETH in their respective portfolios. While USDT offers protection against the sharp downturns experienced in the cryptocurrency markets, the significant returns experienced in general during the sample period mean it is hardly worthwhile including in the optimal portfolio.

\section{Conclusions}

Since its inception, the cryptocurrency market has experienced stunning growth. With increasing participation by institutional investors, the total market value exceeded USD 1 trillion by the start of 2021. We use a DCC-MGARCH model to examine the return and volatility spillovers across three distinct classes of cryptocurrencies: coins, tokens, and stablecoins.

Our results demonstrate that conditional correlations are time-varying and that both $\mathrm{ARCH}$ and GARCH effects play an important role in determining conditional volatility among cryptocurrencies. We find a bi-directional relationship for returns and long-term 
(GARCH) spillovers between BTC and ETH, but only a unidirectional short-term (ARCH) spillover effect from BTC to ETH. We also find spillovers from BTC and ETH to USDT, but no influence running in the other direction, suggesting that USDT does not play an important role in volatility transmission across cryptocurrency markets.

Understanding this interconnectedness is important because it informs investors seeking to diversify their holdings across cryptocurrency classes, since sharp changes in correlation during extreme events (e.g., March 2020) have significant impact on optimal portfolio construction. Certainly, investors should be aware that correlations are time-varying in making investment decisions. In addition, for policy-makers our results provide an indication of potential contagion risk, and suggest continued monitoring of the cryptocurrency market is warranted. For instance, the spillovers we identify indicate potential for contagion among cryptocurrencies and tokens, that could potentially spread to stablecoins.

Future studies could incorporate the use of more advanced methods, such as wavelet analysis or multifractal detrended fluctuation analysis, to uncover the dynamics of spillovers among cryptocurrencies on an intraday basis. It would also be worthwhile considering whether the spillovers to/from stablecoins differs during bull and bear markets once a longer time series of data exists.

Funding: This research received no external funding.

Institutional Review Board Statement: Not applicable.

Informed Consent Statement: Not applicable.

Data Availability Statement: Data and the associated replication code are available in the AARN Cloudstor repository via https:/ / cloudstor.aarnet.edu.au/plus/s/OBSZVKnqtDLaBPk.

Conflicts of Interest: The author declares no conflict of interest.

\section{Notes}

1 See: https://www.economist.com/finance-and-economics/2021/06/10/cryptocoins-are-proliferating-wildly-what-are-theyall-for (accessed on 13 September 2021).

2 Blockchain is a form of decentralised ledger technology that uses strong cryptography to confirm and link data entries. Cryptocurrencies utilise this technology to operate as decentralised virtual currencies, with blockchain thwarting the potential double-spending problem.

3 Source: https:/ / coinmarketcap.com/ (accessed on 12 September 2021).

4 Coinmarketcap has closing prices for Tether from mid-March 2015, and for Ether from September 2015. Our sample starts on 1 January 2016 to coincide with the start of a quarter and ends on 30 June 2021 to coincide with the end of the most recent quarter.

\section{References}

Beneki, Christina, Alexandros Koulis, Nikolaos A. Kyriazis, and Stephanos Papadamou. 2019. Investigating volatility transmission and hedging properties between Bitcoin and Ethereum. Research in International Business and Finance 48: 219-27. [CrossRef]

Bouri, Elie, Brian Lucey, and David Roubaud. 2020. The volatility surprises of leading cryptocurrencies: Transitory and permanent linkages. Finance Research Letters 33: 101188. [CrossRef]

Bouri, Elie, David Gabauer, Rangan Gupta, and Aviral Kumar Tiwarid. 2021a. Volatility connectedness of major cryptocurrencies: The role of investor happiness. Journal of Behavioral and Experimental Finance 30: 100463. [CrossRef]

Bouri, Elie, Luis A. Gil-Alana, Rangan Gupta, and David Roubaud. 2019. Modelling long memory volatility in the Bitcoin market: Evidence of persistence and structural break. International Journal of Finance and Economics 24: 412-26. [CrossRef]

Bouri, Elie, Mahamitra Das, Rangan Gupta, and David Roubaud. 2018. Spillovers between Bitcoin and other assets during bear and bull markets. Applied Economics 50: 5935-49. [CrossRef]

Bouri, Elie, Tareq Saeed, Xuan Vinh Vo, and David Roubaud. 2021b. Quantile connectedness in the cryptocurrency market. Journal of International Financial Markets, Institutions and Money 71: 101302. [CrossRef]

Canh, Nguyen Phuc, Udomsak Wongchoti, Su Dinh Thanh, and Nguyen Trung Thong. 2019. Systematic risk in cryptocurrency market: Evidence from DCC-MGARCH model. Finance Research Letters 29: 90-100. [CrossRef]

Caporale, Guglielmo Maria, Woo-Young Kang, Fabio Spagnolo, and Nicola Spagnolo. 2021. Cyber-attacks, spillovers and contagion in the cryptocurrency markets. Journal of International Financial Markets, Institutions and Money 74: 101298. [CrossRef] 
Engle, Robert F. 1982. Autoregressive Conditional Heteroskedasticity with estimates of the variance of UK inflation. Econometrica 50: 987-1008. [CrossRef]

Engle, Robert F. 2002. Dynamic conditional correlation: A simple class of multivariate generalized autoregressive conditional heteroskedasticity models. Journal of Business E Economic Statistics 20: 339-50.

Gkillas, Konstantinos, and Paraskevi Katsiampa. 2018. An application of extreme value theory to cryptocurrencies. Economics Letters 164: 109-11. [CrossRef]

Härdle, Wolfgang Karl, Campbell R. Harvey, and Raphael CG Reule. 2020. Understanding cryptocurrencies. Journal of Financial Econometrics 18: 181-208. [CrossRef]

Ji, Qiang, Elie Bouri, Chi Keung Marco Lau, and David Roubaud. 2019. Dynamic connectedness and integration in cryptocurrency markets. International Review of Financial Analysis 63: 257-72. [CrossRef]

Katsiampa, Paraskevi, Shaen Corbet, and Brian Lucey. 2019. Volatility spillover effects in leading cryptocurrencies: A BEKK-MGARCH analysis. Finance Research Letters 29: 68-74. [CrossRef]

Koutmos, Dimitrios. 2018. Return and volatility spillovers among cryptocurrencies. Economics Letters 173: 122-27. [CrossRef]

Kroner, Kenneth F., and Jahangir Sultan. 1993. Time dynamic varying distributions and dynamic hedging with foreign currency futures. Journal of Financial and Quantitative Analysis 28: 535-51. [CrossRef]

Kroner, K. F., and V. K. Ng. 1998. Modelling asymmetric movements of asset prices. Review of Financial Studies 11: 817-44. [CrossRef]

Kumar, Anoop S., and Suvvari Anandarao. 2019. Volatility spillover in crypto-currency markets: Some evidences from GARCH and wavelet analysis. Physica A: Statistical Mechanics and its Applications 524: 448-58.

Kyriazis, Nikolaos A. 2019. A survey on empirical findings about spillovers in cryptocurrency markets. Journal of Risk and Financial Management 12: 170. [CrossRef]

Ling, Shiqing, and Michael McAleer. 2003. Asymptotic theory for a vector ARMA-GARCH model. Econometric Theory 19: 278-308. [CrossRef]

Liu, Yukun, and Aleh Tsyvinski. 2021. Risks and returns of cryptocurrency. Review of Financial Studies 34: 2689-727. [CrossRef]

Moratis, George. 2021. Quantifying the spillover effect in the cryptocurrency market. Finance Research Letters 38: 101534.

Naeem, Muhammad Abubakr, Elie Bouri, Zhe Peng, Syed Jawad Hussain Shahzad, and Xuan Vinh Vo. 2021. Asymmetric efficiency of cryptocurrencies during COVID19. Physica A: Statistical Mechanics and its Applications 565: 125562. [CrossRef]

Qureshi, Saba, Muhammad Aftab, Elie Bouri, and Tareq Saeed. 2020. Dynamic interdependence of cryptocurrency markets: An analysis across time and frequency. Physica A: Statistical Mechanics and its Applications 559: 125077. [CrossRef]

Sadorsky, Perry. 2012. Correlations and volatility spillovers between oil prices and the stock prices of clean energy and technology companies. Energy Economics 34: 248-55. [CrossRef]

Sensoy, Ahmet, Thiago Christiano Silva, Shaen Corbet, and Benjamin Miranda Tabak. 2021. High-frequency return and volatility spillovers among cryptocurrencies. Applied Economics 53: 4310-28. [CrossRef]

Smales, Lee A. 2019. Bitcoin as a safe haven: Is it even worth considering? Finance Research Letters 30: 385-93. [CrossRef]

Smales, Lee A. 2020. One cryptocurrency to explain them all? Understanding the importance of Bitcoin in cryptocurrency returns. Economic Papers 39: 118-32. [CrossRef]

Xu, Qiuhua, Yixuan Zhang, and Ziyang Zhang. 2021. Tail-risk spillovers in cryptocurrency markets. Finance Research Letters 38 : 101453. [CrossRef]

Yi, Shuyue, Zishuang Xu, and Gang-Jin Wang. 2018. Volatility connectedness in the cryptocurrency market: Is Bitcoin a dominant cryptocurrency? International Review of Financial Analysis 60: 98-114. [CrossRef] 